\title{
Sistemas de información como soporte para la Acreditación
}

\author{
Information systems as a support for Accreditation
}

\author{
Carmenza Gallego Giraldo \\ Directora Programa de Trabajo Social \\ Universidad de Caldas \\ carmenza.gallego@ucaldas.edu.co
}

\section{Introducción}

Las demandas internas y externas exigen que las Universidades, en particular las Públicas, generen sinergias entre sus sistemas de aseguramiento de la calidad, en tanto posibilitan centrar su atención en lo misional y gestar herramientas integradoras que acotan distancias entre lo que se es y lo que se desea ser. El SIG, es un sistema único de aseguramiento de la calidad, que se referencia en las normas-lineamientosdirectrices- como modelos pero no como fines en sí mismos, por lo que permite construir un cimiento sólido, y a la vez flexible, que permite la incorporación de nuevos requerimientos y demandas del contexto regional, nacional e internacional, en cumplimiento del compromiso de la Universidad con la calidad. El sistema concibe la autoevaluación como una práctica permanente, mediante la cual la Institución auto-reflexiona y mira la forma en que realiza sus diferentes funciones con el propósito de identificar $y$ potenciar sus fortalezas para convertirlas en opciones de desarrollo, al igual que para superar sus debilidades, a través de la ejecución de planes de mejoramiento elaborados a partir del proceso de auto-reconocimiento.

\section{Discusión}

El sistema ha permitido acreditar y reacreditar varios de sus programas académicos, obtener la reacreditación institucional y la certificación de calidad basadas en las normas ISO 9001:2008 y NTCGP 1000:2004 ICONTEC e IQNet- de su Sistema Integrado de Gestión.

Como parte del sistema se abordan los estudios de pertinencia de los programas académicos, se adelantan procesos autónomos de evaluación de departamentos y se gestionan herramientas de calidad tales como: las pruebas de estado Saber Pro, los registros calificados y los procesos de acreditación. A propósito de éstas, tras el levantamiento de los procesos (ver siguiente gráfica y link), se han incorporado compromisos $\mathrm{y}$ acciones de mejora desde las diferentes instancias responsables, con el propósito de garantizar una dinámica permanente de cambio orientado a cualificar los procesos y, por ende, alcanzar los resultados esperados.

\section{Ver procesos:}

http://sig.ucaldas.edu.co/gestionDocumental/ contenidoMapaProcesos.php

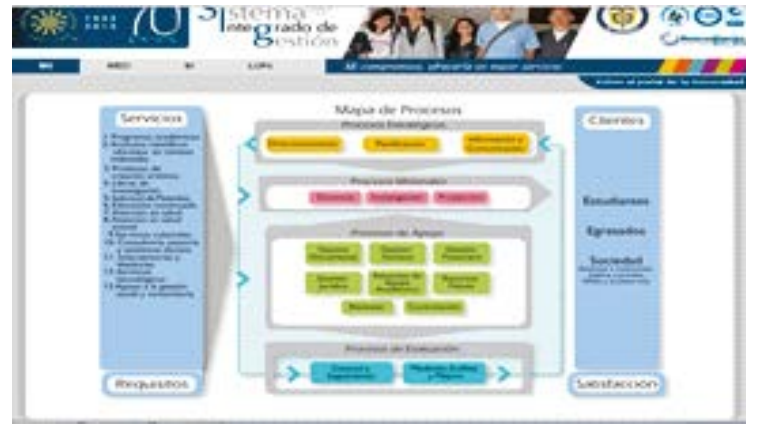

El Sistema también permite la generación de consultas de percepción a la comunidad académica sobre la calidad de los servicios y los diferentes factores y características adoptados por el Consejo Nacional de Acreditación para efecto de la autoevaluación institucional $y$ de programas. Estas consultas se desarrollan mediante encuestas electrónicas (ver siguiente gráfica y link) que son objeto de análisis por parte de la alta dirección y las unidades responsables de los diferentes temas allí indicados, lo cual se convierte en insumo para orientar acciones correctivas, preventivas y de mejora. Además, 
se cuenta con un sistema de información en línea que permite orientar la gestión académica y administrativa, así como monitorear en forma permanente el cumplimiento de las metas, propósitos y procesos institucionales para facilitar los procesos de autoevaluación, planeación, autorregulación y la toma de decisiones al respecto.

Ver: http://sig.ucaldas.edu.co/gestionDocumental/ lupa/principal.php
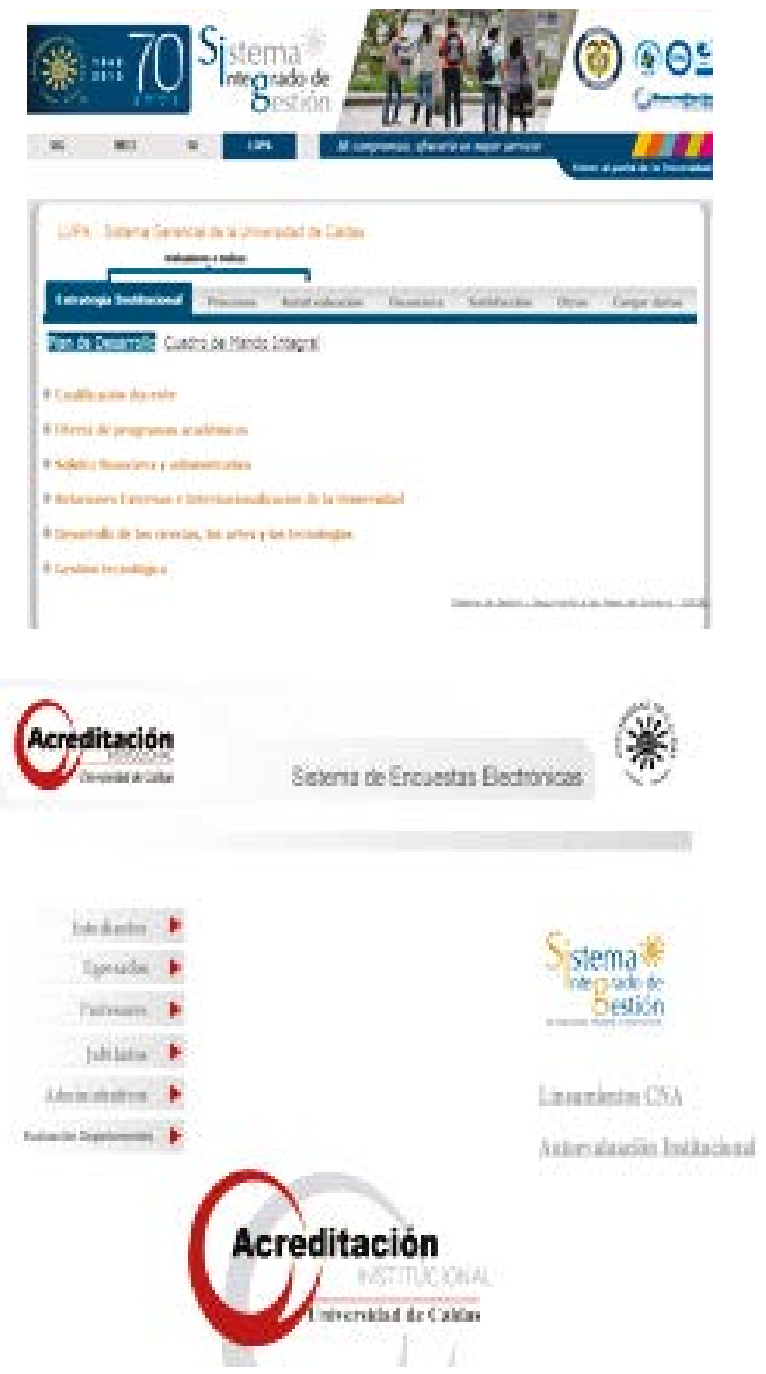

Dicho Sistema de Información alinea la información institucional de tipo estadística, documental y de percepción (o de encuestas) con las metas e indicadores contenidos en el Plan de Desarrollo, los Planes de Acción, los procedimientos y la normatividad institucional. Con ello se busca una mirada integral de la Institución conducente a una gestión informada que opera con procedimientos claramente perfilados, orientados sobre criterios de calidad y eficiencia.

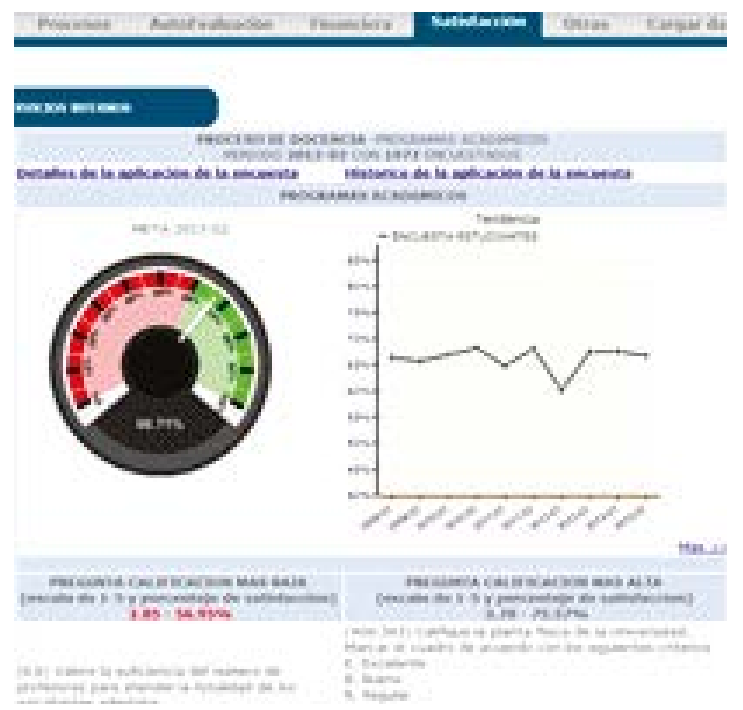

Es claro para la Institución que acreditarse $\mathrm{y}$ certificarse es un compromiso totalmente voluntario y más allá de un logro o reconocimiento, es un reto de mejora continúa con fines netamente sociales y de beneficio público.

El SIG es un sistema unifico de aseguramiento de la calidad en la Universidad de Caldas, mediante el cual se integran los diferentes sistemas de gestión que requieren las instituciones de educación superior para demostrar su calidad integral.

Eldiseño,implementacióny perfeccionamiento del sistema ha surtido las siguientes etapas o fases:

a) Diagnóstico: Sobre la base de los elementos comunes de los diferentes sistemas de gestión de la calidad, se identificaron los aspectos críticos a intervenir, integrándolos a las necesidades de mejoramiento derivadas de los informes de autoevaluación con fines de acreditación institucional y el informe de los pares externos que realizaron dicho proceso.

b) Planificación: Se determinaron las actividades para subsanar las diferencias entre lo que tiene la Universidad y lo que debe tener según los resultados derivados del diagnóstico. 
El producto resultante es un plan detallado de actividades con sus responsables, tiempos de ejecución y plazos.

c) Sensibilización y capacitación: Establecida la base fundamental del proyecto como facilitador del proceso de transformación cultural, era necesario trabajar primero en el componente humano mediante jornadas de sensibilización y capacitación que abordaran el desarrollo de competencias en el saber-ser (calidad como estilo de vida, las interacciones claves para los sistemas de gestión, entre otros), el saber-saber (Sistema Nacional de Aseguramiento de la Calidad - CNA-, Auditorías internas, referentes normativos NTCGP1000:2004, Modelo Estándar de Control Interno,) y el saberhacer (Operación de la herramienta informática Software SIG, construcción de documentos -caracterizaciones, procedimientos, instructivos, guías, trámites-, entrenamiento en el cargo y establecimientos de cadenas de valor), por lo que se desarrollaron diferentes diplomados y cursos con la participación de directivos, docentes y administrativos.

d) Diseño e implementación: Se establece la concepción praxiológica del sistema y se explicita la teleología del mismo, soportadas en las directrices institucionales internas, entre otras, los Acuerdos 027 diciembre de 2004 del Consejo Superior a través del cual se evidencia el compromiso de la Universidad con la calidad en el desarrollo de las funciones sustantivas y el 011 de 2008 del ConsejoSuperior en el cual se integran los diferentes sistemas de aseguramiento de la calidad, tanto administrativa como académica, como se ilustra en la figura 1 y la integración de las herramientas informáticas donde proveen la información necesaria tanto para los aspectos de calidad requeridos en las autoevaluaciones como para la toma de decisiones de los órganos competentes, figura 2.

e) Evaluación: Como mecanismos de evaluación se utilizaron:

- Visita de pares académicos

- Medición de indicadores

- Medición de la satisfacción interna y externa

- Administración del riesgo
- Auditorías internas y externas

- Revisión por la dirección

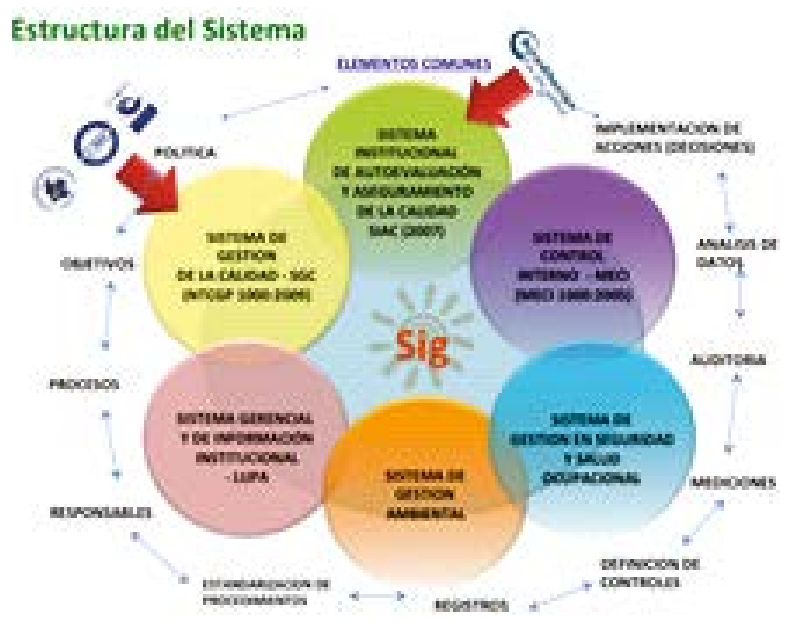

Figura 1.

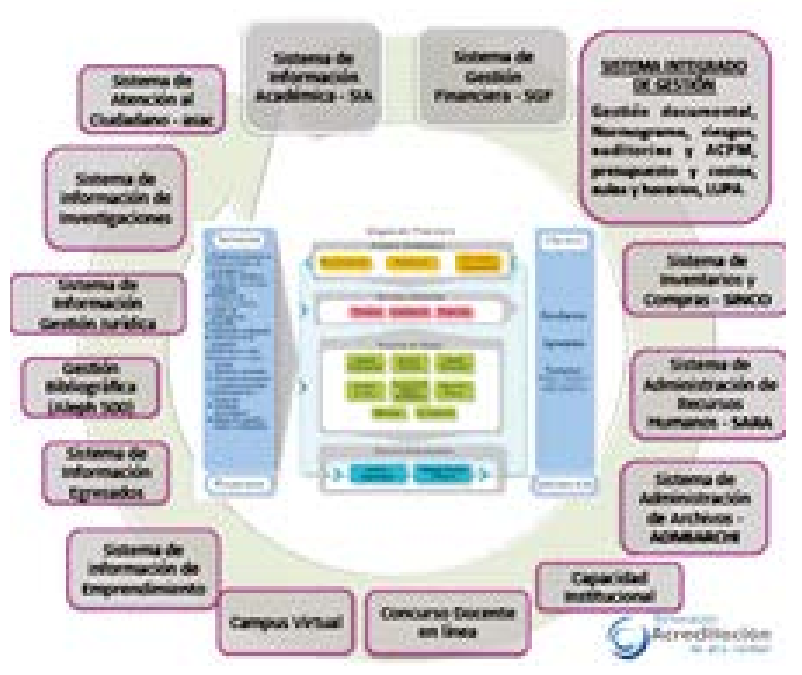

Figura 2.

f) Mejora: Surtidas cada una de las etapas de implementación y fruto delos resultados dela fase de evaluación, se formularon e implementaron las acciones correctivas, preventivas y de mejora necesarias.

g) Consolidación del Sistema: Actualmente el SIG responde a los requisitos de la reacreditación institucional, la acreditación y reacreditación de programas académicos y auditoría para certificación bajo el modelo de gestión de la calidad. 
La posibilidad de ser replicada la experiencia en otras IES es alta sobre todo para las universidades públicas, en tanto la articulación de las herramientas de gestión: Sistema de Gestión de la Calidad bajo la norma NTCGP 1000:2004, Modelo Estándar de Control Interno bajo el modelo MECI 1000:2005, Sistema de Desarrollo Administrativo -SISTEDA y el Sistema de Autoevaluación y Aseguramiento de la Calidad-CNA, son posibles, dado que sus fines, objetivos y estrategias guardan relación y pueden alcanzarse a través de la integración en un solo modelo de gestión.

Con respecto a los recursos económicos, financieros, de infraestructura, tecnológicos y humanos requeridos para la puesta en marcha del Sistema, están centrados sobre todo en inversiones en sistemas de información que apoyen y agilicen la toma de decisiones, en la capacitación de la comunidad universitaria y en la coordinación de acciones en términos de la conformación de equipos de trabajo que entiendan las cadenas de valor de todos los mejoramientos institucionales requeridos.

Frente a los efectos positivos, se puede demostrar, con evidencias, logros relativos a:

- Uso de los datos y las mediciones para el análisis de situaciones a nivel del equipo directivo, soportando la toma de decisiones.

- Involucramiento del Consejo Superior, quien a través del Acuerdo 011 de 2008 establece roles, responsabilidades, compromisos y legitima el Sistema como herramienta de gestión al disponer que toda la documentación aprobada es de fuerza vinculante, es decir, de obligatorio cumplimiento en la Universidad.

- Articulación del sistema de acreditación institucional y de programas, gestión de la calidad y control interno, sirviendo como referente (modelo) para otras Instituciones de Educación Superior.

- Operación de un sistema de evaluación y seguimiento institucional por medio de la medición de indicadores, auditorías internas, medición de la satisfacción y revisión por la dirección.

- Monitoreo permanente del desempeño de la Universidad a través del Sistema Gerencial LUPA.

- Transición hacia una gestión preventiva a través de la administración del riesgo.

- Sistema registrado en el Banco de Éxito del Departamento Administrativo de la Función Pública.

- La reacreditación institucional y la reacreditación y acreditación de los programas académicos. Actualmente se cuenta con el $44 \%$ de los programas académicos universitarios acreditables, acreditados, una línea de base para la acreditación de postgrados y en trámite ante el CNA 6 programas académicos universitarios.

- El 100\% de los programas académicos cuentan con la información institucional y la apreciación de calidad de la comunidad académica disponible para sus procesos de autoevaluación.

Con respecto a los beneficios generados, analizando el "Uso de las tecnologías - TIC's", el resultado verificable es la herramienta que se encuentra ubicada en la página web de la institución en el enlace Sistema Integrado de Gestión, el cual no se requiere clave para acceder; solamente se debe acreditar con un login y clave para algunos roles específicos establecidos.

El seguimiento y evaluación del sistema es permanente en tanto las autoevaluaciones como insumos para sus procesos de aseguramiento de la calidad, ya sean para registro calificado, acreditación, apertura de nuevas cohortes, son requisitos fundamentales para alcanzar sus fines.

De igual manera, las visitas de los pares académicos, las auditorías de seguimiento anual del sistema de gestión de la calidad efectuada por los entes certificadores, las auditorías internas de calidad a los 16 procesos 
y las auditorías de control estatal junto con la revisión anual por la dirección y la rendición de cuentas institucional, son los activadores por excelencia del mejoramiento del SIG, dado que sus hallazgos son llevados a acciones de mejoramiento y ubicados en los planes de acción de las dependencias correspondientes, los cuales deben guardar relación con las variables del plan de desarrollo y con el plan de acción y de mejoramiento institucional.

Como todo ciclo de mejoramiento el sistema ha identificado algunos puntos críticos que requieren ser abordado paulatinamente, entre ellos las entendibles barreras de aprendizaje individual y organizacional. Las primeras, fruto de experiencias y vivencias personales generadas a lo largo de los planes de carrera, las funciones ejercidas y los procesos formativos previos de los funcionarios de la universidad. Las segundas, por tradiciones organizativas centradas en esquemas jerárquicos por dependencias o áreas, rotación de directivos y cambios abruptos en las iniciativas administrativas y los estilos de dirección.

Otras barreras, como la falta de afinidad y usabilidad de los sistemas informáticos, la existencia de una cultura centrada en evidencias impresas, la informalidad en la gestión, la baja utilización de herramientas soportadas en TIC's y una marcada separación entre lo académico y administrativo, han sido identificadas a lo largo de la experiencia.

Delos principales aprendizajes se han inferido:

La coordinación de acciones, el trabajo reflexivo de aprendizaje, la integración del sistema socio - técnico, la generación de leguajes y esquemas de trabajo compartidos, las cadenas de valor, la comprensión de los requerimientos y requisitos de los usuarios, los momentos de verdad y los esquemas de control, son las principales estrategias de trabajo para en el Sistema Integrado de Gestión.

El Sistema no nace en el momento de su implementación, él recupera de la Universidad sus tradiciones e historias y sus modos de operación, convirtiéndolos en diálogos y contextos de calidad al traducirlos en esquemas universales, amigables, comunicables, trazables, medibles y mejorables.

Para hacerlo sostenible, el sistema requiere de una fuerte articulación con las dinámicas y evoluciones de los contextos, con los planes estratégicos institucionales, con las decisiones y compromisos de las directivas y con indicadores que hagan visible sus impactos o beneficios en el tiempo.

Frente al desarrollo de nuevas o mejores prácticas, el sistema permite que cada uno de los funcionarios públicos pueda identificar claramente su propia cadena de valor del trabajo, en tanto visualiza las actividades, tiempos y evidencias de su desempeño a lo largo de los procesos institucionales, soportados en la estandarización y documentación de las actividades que implican y sus mecanismos de control, seguimiento y mejora.

\section{Conclusiones:}

La Universidad de Caldas fue la cuarta universidad del País que diseñó e implementó un sistema integral de aseguramiento de la calidad soportada en un direccionamiento del Consejo Superior que soporta su permanencia en el tiempo, sistema que fue inscrito en el banco de éxitos de la Función Pública lo que le permite ser replicado en cualquier institución que desea aplicarlo.

Este Sistema, articulado y flexible, permite responder a los posibles cambios que puedan ocurrir en materia de direccionamiento institucional, directivas universitarias, legislación y normativa interna y externa, demandas del contexto, entre otros, en tanto se sustenta en un ciclo de intervención social donde la reflexión pública, la planificación conjunta y la acción coordinada son sus pilares fundamentales.

No se requiere para su implementación la creación de unidades de gestión que amplíen las estructuras funcionales, dado que éste se ha entendido como parte de las responsabilidades 
de todos los funcionarios de la institución, de manera muy especial para los cargos de alto nivel, tales como Rector, Vicerrectores, Secretario General y Jefes de Oficina, quienes se constituyen en 11 líderes de procesos. Es requisito, para facilitar el conocimiento inicial requerido y el cumplimiento de las responsabilidades frente al sistema, que la inducción, la reinducción y el entrenamiento de todos los funcionarios incluya como tema esencial el Sistema.

Finalmente, la posibilidad de ser replicada la experiencia en otras IES es alta sobre todo para las públicas en tanto la articulación de las herramientas de gestión: Sistema de Gestión de la Calidad bajo la norma NTCGP 1000:2004, Modelo Estándar de Control Interno bajo el modelo MECI 1000:2005, Sistema de Desarrollo Administrativo -SISTEDA y el Sistema de Autoevaluación y Aseguramiento de la CalidadCNA, son posibles, en tanto sus fines, objetivos y estrategias guardan relación y pueden alcanzarse a través de la integración en un solo modelo de gestión.

\section{Referencias}

Norma técnica de Calidad en la Gestión Pública. NTCGP 1000:2009
Modelo Estándar de Control Interno para el Estado Colombiano. MECI 1000:2005.

Decreto 2482 de 2012 Nivel Nacional. Sistema de Desarrollo Administrativo - SISTEDA.

Silva, J., Bernal, Elizabeth. Caracterización de tres modelos de aseguramiento interno de la calidad a partir de la experiencia de las IES en Colombia. ISBN 978-958-691-6134.2014

Estado del arte del Sistema Nacional de Acreditación e identificación de rutas y tópicos de investigación y profundización de calidad. Grupo de Investigación en Evaluación UNAL, 2013.

Nuevos Lineamientos para la Acreditación Institucional. 2015

Nuevos Lineamientos Acreditación de Programas de Pregrado. 2013

Lineamientos para la Acreditación de Alta Calidad de Programas de Maestría y Doctorado. 2010. 